\title{
Metformin in patients with type 2 diabetes mellitus and heart failure: a review
}

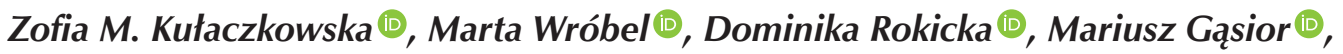 \\ Krzysztof Strojek ${ }^{\circledR}$

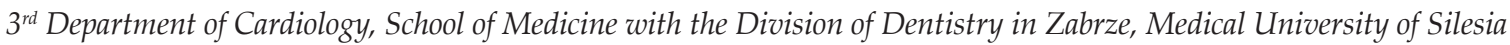 \\ in Katowice, Silesian Centre for Heart Diseases, Zabrze, Poland
}

\begin{abstract}
Diabetes mellitus is a major, global problem. Among the numerous complications of diabetes, there is increasing concern over the coexisting heart failure. Metformin is the most frequently used oral antidiabetic drug that is considered to be safe and effective in the management of type 2 diabetes mellitus. Since the publication of the UK Prospective Diabetes Study, it has been suggested that metformin might improve cardiovascular prognoses. Results from available studies have shown that metformin therapy in patients with type 2 diabetes mellitus and heart failure was associated with improved clinical outcomes when compared with other oral antidiabetic agents, insulin, or lifestyle management. However, there have been no randomized controlled trials evaluating the influence of metformin use on clinical outcomes in patients with type 2 diabetes mellitus and heart failure. New evidence from large cardiovascular outcome trials that showed a reduction in heart failure hospitalization for SGLT2 inhibitors caused changes in recommendations on the management of hyperglycaemia. Currently, the European Society of Cardiology recommends sodium-glucose co-transporter 2 inhibitors in patients with type 2 diabetes mellitus and heart failure or at high risk for heart failure, as a first choice in drug naïve patients, or as a second drug if the patient is already on metformin. The aim of our study is to review the current state of knowledge about the position of metformin in the treatment of patients with type 2 diabetes mellitus and heart failure. (Endokrynol Pol 2021; 72 (2): 163-170)
\end{abstract}

Key words: metformin; type 2 diabetes mellitus; heart failure

\section{Introduction}

Diabetes mellitus is a significant medical, social, and economic problem. Data from the International Diabetes Federation show that diabetes affects 463 million adults worldwide, and it is estimated that one in two people living with diabetes are unaware of their condition [1]. In Poland, the number of people suffering from diabetes amounted to 2.533 million in 2017 [2]. Among the numerous health complications of diabetes mellitus there is increasing concern over a previously undervalued issue: coexisting heart failure (HF).

In 2020 , on the $56^{\text {th }}$ annual meeting of the European Association for the Study of Diabetes (EASD), the CAPTURE study on the prevalence of cardiovascular disease (CVD) involving 9823 patients with type 2 diabetes mellitus (T2DM) from 13 countries was presented. Overall, $34.8 \%$ of participants had CVD, and $2.4 \%$ suffered from $\mathrm{HF}$ [3]. In the analysis from the CVD-REAL 2 multinational cohort study on the risk of cardiovascular (CV) events and death in 38,6248 adult patients with T2DM, newly initiated on sodium-glucose co-transporter 2
(SGLT2) inhibitors or dipeptidyl peptidase-4 (DPP-4) inhibitors, HF was present in $7 \%$ of patients at baseline [4]. The prevalence of HF in diabetic patients in real-world studies ranges from 5 to $6.8 \%[5,6]$.

Metformin is the most frequently used oral antidiabetic drug that is considered to be safe and effective in the management of T2DM [7]. It acts as a glucose-lowering agent through the decrease of hepatic glucose production, as well as lowering insulin resistance in peripheral tissues. Besides its neutral effect on body weight and its positive impact on lipidogram, metformin's influence on the CV system seems to be cardioprotective, because it has a beneficial impact on the vascular wall and clotting system parameters [8]. Since the publication of UK Prospective Diabetes Study 34 (UKPDS 34), metformin has emerged as a drug that seems to decrease the risk of diabetes-related endpoints, including macrovascular and microvascular complications, in overweight patients with T2DM. The results of UKPDS 34 showed that patients who were allocated metformin had a $32 \%$ lower risk $(\mathrm{p}=0.0023)$ of developing any diabetes-related endpoint, a $36 \%$ lower risk 
$(\mathrm{p}=0.011)$ of all-cause mortality, and a 39\% lower risk $(\mathrm{p}=0.010)$ of myocardial infarction (MI), in comparison to management with diet alone [9]. Moreover, in the post-trial 10-year follow-up of intensive glucose control, metformin proved to have a long-term effect on macrovascular outcomes, decreasing the risk of MI by $33 \%$ ( $p=0.005$ ) [10].

New evidence from large cardiovascular outcome trials (CVOTs), which showed CV benefits from the use of newer glucose-lowering drugs in patients with CVD or at very high/high CV risk, led to changes in the recommendations on the management of hyperglycaemia.

The aim of this article is to review the current state of knowledge about the position of metformin in the treatment of patients with T2DM and HF.

\section{Metformin in patients with type 2 diabetes mellitus and heart failure: a review of research studies}

In the analysis of 1833 new users of oral antidiabetic agents with incident HF, metformin use, in monotherapy $(n=208)$ or in combination with sulfonylureas $(\mathrm{n}=852)$, was compared with sulfonylurea monotherapy $(\mathrm{n}=773)$ (Tab. 1). During the 2.5-year follow-up, all-cause mortality and all-cause hospitalization, both at 1 year and at the end of the follow-up period, were evaluated. Metformin, alone or in combination with sulfonylureas, was associated with reduced 1-year and longer-term all-cause mortality in comparison to sulfonylurea monotherapy. On the other hand, there was no significant association between compared groups and all-cause hospitalization. In the composite outcome analysis fewer deaths and/or hospitalizations occurred in patients on metformin monotherapy and combination therapy when compared with sulfonylurea monotherapy [11].

Sulfonylurea monotherapy was also used as a reference group in the observational study of 10,920 patients treated with metformin, sulfonylureas, and/or insulin, and hospitalized for the first time for HF. Metformin in monotherapy and in combination with sulfonylureas was associated with lower all-cause mortality compared with sulfonylureas in monotherapy. The results were similar in a separate analysis of patients using and not using insulin [12].

In the observational study of 16,417 patients with T2DM, discharged from a hospital with a major discharge diagnosis of $\mathrm{HF}$, the influence of insulin-sensitizing drugs on 1-year all-cause mortality, 1-year all-cause hospitalization, and HF hospitalization was assessed. Individuals treated with thiazolidinediones $(n=2226)$, metformin ( $\mathrm{n}=1861)$, as well as both thiazolidinediones and metformin ( $n=261$ ) had a lower risk of death com- pared with patients not treated with insulin-sensitizing drugs $(n=12069)$. There was no difference in all-cause hospitalization between insulin-sensitizing agents, although patients receiving metformin had a lower risk of hospitalization for $\mathrm{HF}$, while patients receiving thiazolidinediones had a higher risk of hospitalization for HF when compared with the therapy without an insulin-sensitizing drug [13].

MacDonald et al. designed a case-control study that assessed treatment with metformin in patients newly diagnosed with HF and T2DM, during a median follow-up of 2.8 years. A comparison group consisted of individuals without an antidiabetic drug in their therapy. Metformin monotherapy was associated with lower all-cause mortality when compared with patients not exposed to antidiabetic therapy [14].

In the analysis of 6185 ambulatory patients with $\mathrm{HF}$ and T2DM, 1561 participants treated with metformin were compared with 4624 patients not treated with metformin. During the follow-up period of 2 years, the risk of death and the risk of hospitalization were assessed. Additionally, the relationship between metformin use and outcomes (time to death, time to HF hospitalization, and time to any hospitalization) was assessed using propensity score-matched analysis that consisted of 29 baseline variables, and compared patients receiving metformin with patients not receiving metformin. Metformin therapy was associated with reduced mortality in comparison to therapy without metformin, in both unadjusted and propensity score-matched analysis. The risk of hospitalization for $\mathrm{HF}$ as well as all-cause hospitalization was lower in patients receiving metformin compared with those not receiving metformin in unadjusted analysis; however, in propensity score-matched analysis there was no statistically significant difference between groups [15].

Shah et al. investigated the use of metformin in patients with left ventricular ejection fraction (LVEF) below 40\% (mean LVEF $24 \pm 7 \%$ ) and T2DM. Forty-two per cent of patients were in New York Heart Association (NYHA) class III and 45\% in NYHA class IV. Ninety-nine patients who were on metformin therapy, as a monotherapy or in combination with other antidiabetic drugs, were compared with 302 patients using oral antidiabetic drugs other than metformin and/or insulin. During the 6-month follow-up period, LVEF significantly improved in patients on metformin therapy compared with patients without metformin in their therapy. However, after adjustment for angiotensin-converting enzyme inhibitors (ACEI)/angiotensin receptor blockers (ARB) and B-blocker therapy the improvement in LVEF was nonsignificant. Patients on metformin therapy had significantly longer survival and lower risk of combined endpoint (all-cause mortality and the need for urgent 


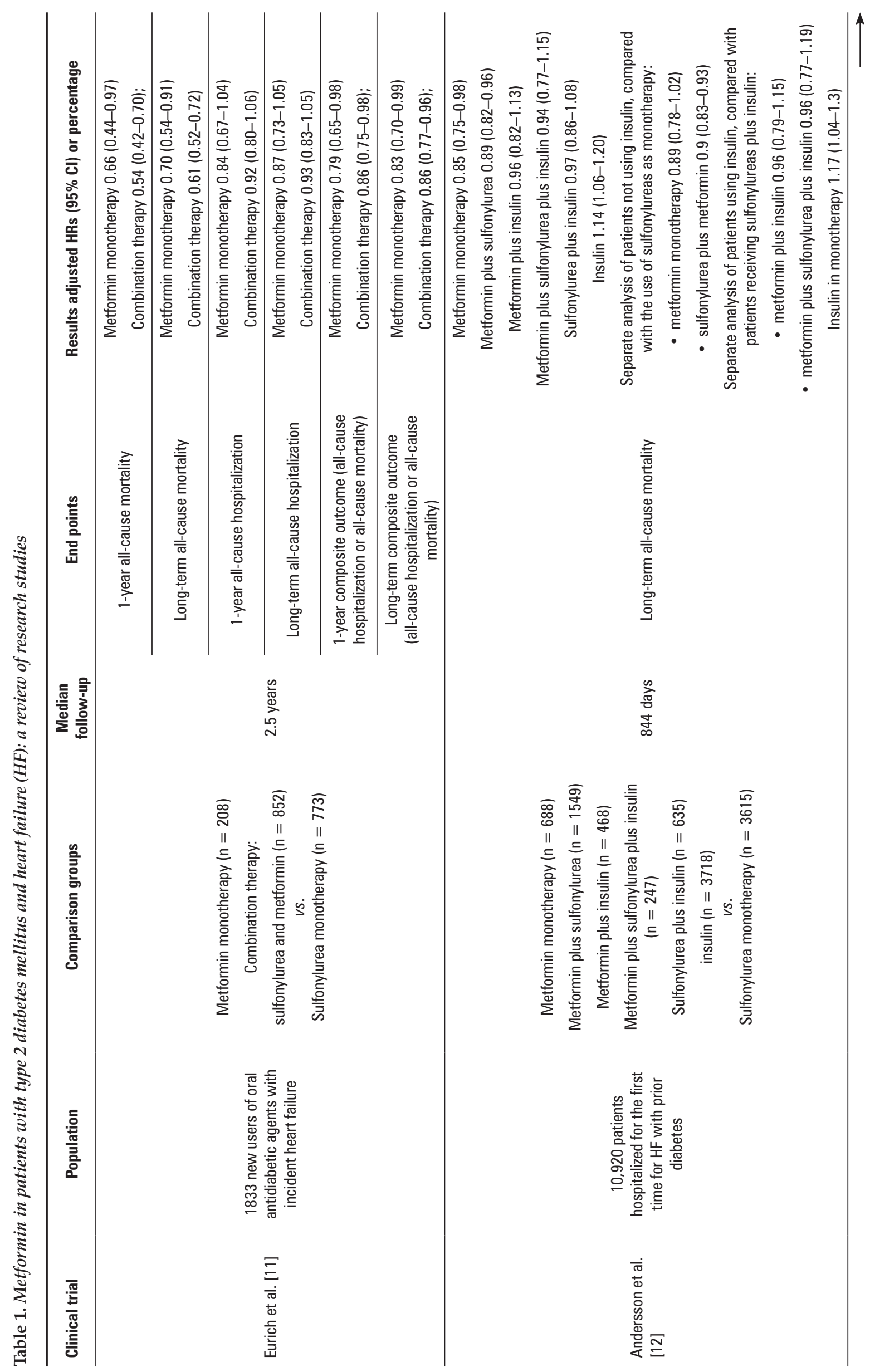




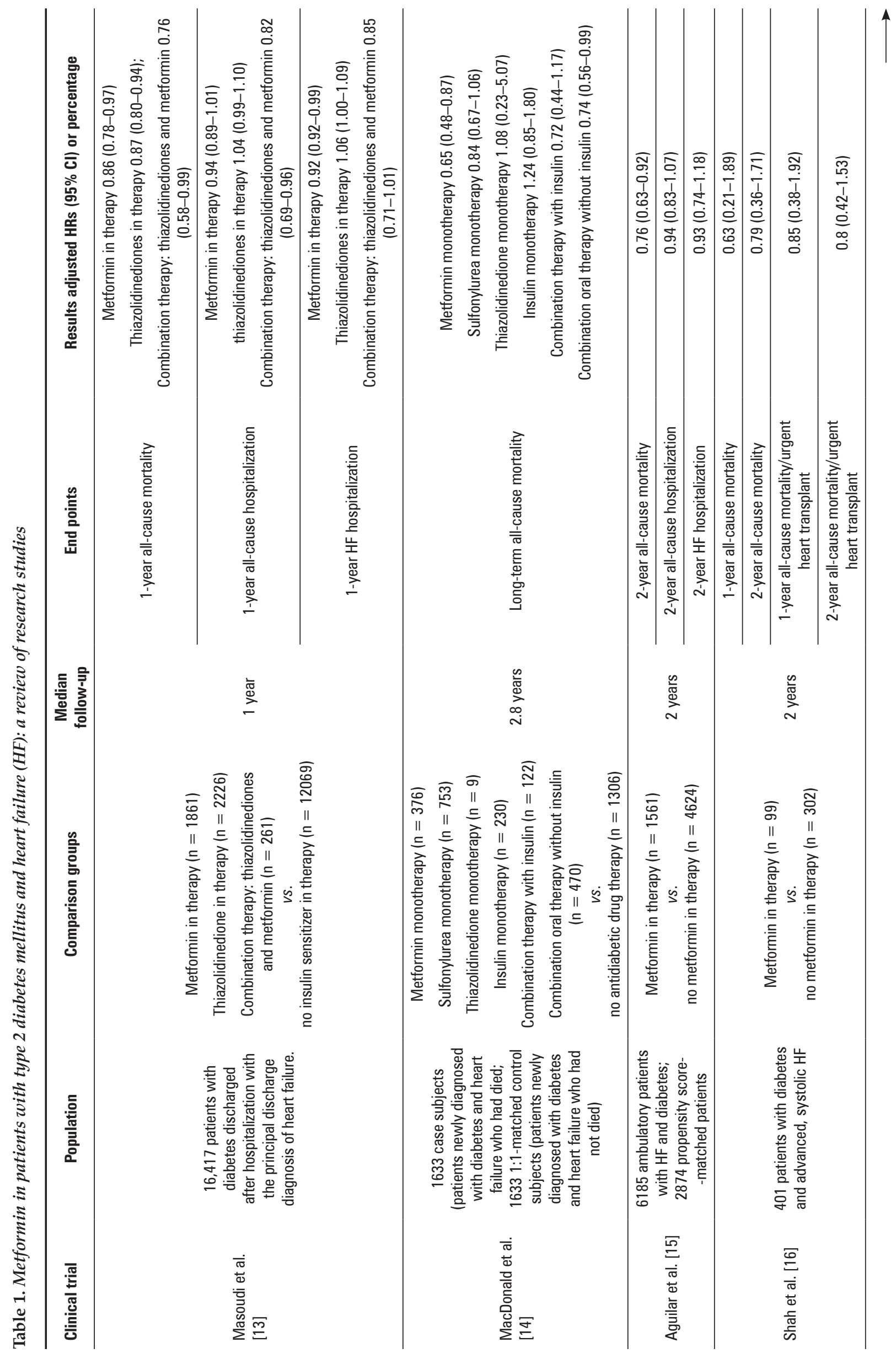


heart transplant) compared with patients without metformin in their therapy. In multivariate analysis there was no significant difference in survival between the analysed groups [16].

In the analysis from the PL-ASC registry (Polish Registry of Acute Coronary Syndromes), diabetic patients after acute coronary syndrome treated with percutaneous coronary intervention with no history of prior CVD were assessed. Patients treated with metformin were compared with patients not treated with metformin, before admission to a hospital. LVEF at discharge from the hospital was evaluated. The number of patients with LVEF below $40 \%$ was significantly lower in patients on metformin therapy in comparison to patients receiving antidiabetic drugs other than metformin (12\% vs. $17 \%$, $\mathrm{p}<0.001$ ) [17].

The impact of metformin use on patients with T2DM discharged from a hospital with a major diagnosis of acute HF was assessed in 835 participants. During a mean follow-up period of 2.4 years, long-term all-cause mortality was significantly lower in patients treated with metformin in comparison to patients without metformin in their therapy. In the multivariate analysis, metformin use was also significantly associated with lower all-cause mortality rates [18].

\section{Metformin in patients with type 2 diabetes mellitus and heart failure: a review of guidelines}

In the position statement from the Heart Failure Association (HFA) of the European Society of Cardiology (ESC) on type 2 diabetes mellitus and heart failure, metformin was presented as an antidiabetic drug that might be safe in heart failure and could be recommended as a first-line therapy for patients with T2DM and $\mathrm{HF}$, who have preserved or moderately reduced renal function. However, the document emphasizes the lack of randomized controlled trials of metformin use in patients with T2DM and HF [19].

According to the 2019 ESC Guidelines on diabetes, pre-diabetes, and cardiovascular diseases developed in collaboration with the EASD, metformin is safe at all stages of HF, reducing the risk of death and HF hospitalization in comparison to insulin and sulfonylureas. In the previous guidelines (2013) metformin was considered as a first-line therapy in patients with T2DM, independently of the patient's cardiovascular profile [20]. However, there have been changes in the recommendations due to the recent publication of several CVOTs that indicate CV benefit from the use of glucagon-like peptide-1 (GLP-1) receptor agonists and SGLT2 inhibitors in patients with T2DM and CVD or those at very high/high CV risk. As a consequence, 
the current guidelines suggests that the choice of antidiabetic drug in patients with T2DM should depend on the presence of CVD and CV risk. Thus, patients with T2DM and prevalent CVD or with very high/high $\mathrm{CV}$ risk should receive drugs with proven $\mathrm{CV}$ benefit, GLP-1 receptor agonists, or SGLT2 inhibitors, as a first choice in drug-naïve patients or as a second drug if the patient is already on metformin. On the other hand, in patients with T2DM without CVD or at moderate CV risk, metformin should be recommended as first-line therapy, especially in overweight individuals. In regard to the selection of glucose-lowering agents in patients with T2DM and HF or at high risk for HF, metformin and SGLT2 inhibitors are the first-line therapy. SGLT2 inhibitors are recommended for the treatment of patients with T2DM and HF (class I of recommendation) because they seem to reduce HF-related endpoints, while metformin should be considered in these patients (class IIa of recommendation), which is based on observational studies and everyday clinical practice [21].

New evidence from CVOTs has also implied an important change in the 2018 Consensus Report by the American Diabetes Association (ADA) and the EASD on the Management of Hyperglycaemia in Type 2 Diabetes. Since SGLT2 inhibitors have been proven to reduce hospitalization for HF in patients with atherosclerotic cardiovascular disease in comparison to placebo, and they are recommended in patients with T2DM and coexisting HF or at risk for HF, as a part of glucose-lowering treatment. Nevertheless, metformin remains the preferred initial medication for the management of T2DM unless it is contraindicated or not tolerated. Subsequently, if $\mathrm{HbA}_{1 \mathrm{c}}$ is above the target and HF predominates, a SGLT2 inhibitor with evidence of reducing HF progression should be added to the therapy [22].

Furthermore, in 2019 the Consensus Report on the Management of Hyperglycaemia in Type 2 Diabetes was updated by the ADA and the EASD, as a consequence of new research findings. The updated report suggests considering an initial combination therapy, composed of metformin and SGLT2 inhibitor, in new-onset diabetes mellitus if HF coexists, independently of baseline $\mathrm{HbA}_{1 \mathrm{c}}$ or individualized $\mathrm{HbA}_{1 \mathrm{c}}$ target, due to reduced hospitalization for $\mathrm{HF}$, major adverse cardiovascular events, and cardiovascular death, especially in patients with reduced left ventricular ejection fraction $(\mathrm{LVEF}<45 \%)$ [23].

\section{Discussion}

The impact of metformin therapy on clinical outcomes in patients with T2DM and coexisting HF has been assessed in large observational studies. In this review, 8 observational studies were included (Table 1). Despite an extensive literature search, no randomized controlled trials evaluating the effects of metformin therapy in patients with T2DM and HF were identified. Indeed, the execution of a randomized controlled trial could be difficult because of the common use of metformin in patients with HF $[24,25]$. For many years metformin was contraindicated in patients with HF due to the risk of metformin-associated lactic acidosis. However, the incidence of lactic acidosis in clinical practice has proved to be very low and the United States Food and Drug Administration has removed congestive HF from the boxed warning section of metformin [26]. Nevertheless, hypoxic states such as the setting of acute congestive $\mathrm{HF}$ remain contraindications for metformin use, especially when hypoperfusion and hypoxaemia coexist [27]. At the same time, in the Polish Summary of Product Characteristics, HF is consistently one of the main contraindications of metformin, although it should be assumed that this refers to acute states [28].

Data from available studies have shown that metformin use, assessed in monotherapy or in combination with other oral antidiabetic drugs and/or insulin, in patients with T2DM and HF, was associated with improved clinical outcomes when compared with other oral antidiabetic agents, insulin, or lifestyle management. Based on this result, metformin is considered to be a safe drug in heart failure and remains the first-line treatment in patients with heart failure and diabetes, which is supported by clinical experience [22]. However, the safety of metformin in the results of observational studies might be a consequence of its cautious use in patients with HF due to its contraindications. Additionally, when metformin is compared to other antidiabetic agents, there is a probability that the outcomes are the consequence of a harm effect of the comparator agent. This is the reason why there is a need for randomized controlled trials to show not only the safety of metformin but also its beneficial effect on the onset and the course of heart failure.

SGLT2 inhibitors have emerged as antidiabetic drugs that significantly reduce HF hospitalization and $\mathrm{CV}$ death in patients with T2DM and high CV risk, when compared with placebo in large CVOTs $[29,30]$. Moreover, trials on dapagliflozin and empagliflozin showed that SGLT2 inhibitors reduce HF hospitalization and CV death in patients with HF, not only when diabetes coexists but also in patients without diabetes [31]. Therefore, the position of metformin in the treatment of patients with T2DM and HF has changed. Currently, ESC Guidelines recommend SGLT2 inhibitors in patients with T2DM and HF or at high risk for $\mathrm{HF}$, as a first choice in drug-naïve patients or as a second drug if the patient is already on metformin [21]. However, 
this statement is not confirmed by any randomized controlled clinical trial, and it has not been proven that starting glucose-lowering treatment with SGLT2 inhibitors, instead of metformin, is beneficial. In CVOTs, SGLT2 inhibitors were added to the standard therapy in which more than $70 \%$ of patients had already received metformin at baseline. Additionally, hospitalization due to HF was a secondary endpoint in each trial; thus, groups of patients with HF were not thoroughly characterized at baseline and were relatively small. Finally, there are no clinical trials on SGLT2 inhibitors that show a reduction of $\mathrm{CV}$ risk in patients with $\mathrm{HbA}_{1 \mathrm{c}}<7 \%$, because all of the participants of CVOTs had $\mathrm{HbA}_{1 \mathrm{c}}>7 \%$ at baseline [22].

\section{Conclusions}

According to the Summary of Product Characteristics, metformin is contraindicated in patients with HF. However, metformin is the most frequently used oral antidiabetic drug in T2DM; thus, a great number of patients with HF receive metformin despite the contraindications. The analysis of data from observational studies and meta-analyses shows that metformin has a favourable effect in patients with T2DM and HF. Modification of the Summary of Product Characteristics should be considered after performing randomized controlled trials on metformin treatment in patients with T2DM and HF

\section{Conflict of interest}

The authors certify that they have no affiliations with or involvement in any organization or entity with any financial interest (such as honoraria, educational grants, participation in speakers' bureaus, membership, employment, consultancies, stock ownership, or other equity interest, expert testimony, or patent-licensing arrangements), or non-financial interest (such as personal or professional relationships, affiliations, knowledge, or beliefs) in the subject matter or materials discussed in this manuscript.

\section{References}

1. Saeedi P, Salpea P, Karuranga S, et al. IDF Diabetes Atlas Committee. Global and regional diabetes prevalence estimates for 2019 and projections for 2030 and 2045: Results from the International Diabete Federation Diabetes Atlas, 9 edition. Diabetes Res Clin Pract. 2019; 157: 107843, doi: 10.1016/j.diabres.2019.107843, indexed in Pubmed: 31518657

2. Topor-Madry R, Wojtyniak B, Strojek K, et al. Prevalence of diabetes in Poland: a combined analysis of national databases. Diabet Med. 2019; 36(10): 1209-1216, doi: 10.1111/dme.13949, indexed in Pubmed: 30889281.

3. Mosenzon O, Alguwaihes A, Arenas Leon JL et al. CAPTURE: a cross-sectional study of the contemporary (2019) prevalence of cardiovascular disease in adults with type 2 diabetes across 13 countries. Poster presented at: 56th Annual Meeting of the European Association for the study of diabetes; September 21-25, 2020; Virtual meeting. 2019.

4. Kohsaka S, Lam CSP, Kim DJ, et al. CVD-REAL 2 Investigators and Study Group. Risk of cardiovascular events and death associated with initiation of SGLT2 inhibitors compared with DPP-4 inhibitors: an analysis from the CVD-REAL 2 multinational cohort study. Lancet Diabetes Endocrinol. 2020; 8(7): 606-615, doi: 10.1016/S2213-8587(20)30130-3, indexed in Pubmed: 32559476.

5. Kosiborod M, Lam CSP, Kohsaka S, et al. CVD-REAL Investigators and Study Group. Cardiovascular Events Associated With SGLT-2 Inhibitors Versus Other Glucose-Lowering Drugs: The CVD-REAL 2 Study. J Am Coll Cardiol. 2018; 71(23): 2628-2639, doi: 10.1016/j.jacc.2018.03.009, indexed in Pubmed: 29540325.

6. Birkeland KI, Jørgensen ME, Carstensen B, et al. Cardiovascular mortality and morbidity in patients with type 2 diabetes following initiation of sodium-glucose co-transporter-2 inhibitors versus other glucose-lowering drugs (CVD-REAL Nordic): a multinational observational analysis. Lancet Diabetes Endocrinol. 2017; 5(9): 709-717, doi: 10.1016/S2213-8587(17)30258-9, indexed in Pubmed: 28781064.

7. Araszkiewicz A, Bandurska-Stankiewicz E, Budzyński A, et al. 2019 Guidelines on the management of diabetic patients. A position of Diabetes Poland. Clin Diab. 2019; 8(1): 1-95, doi: 10.5603/dk.2019.0001.

8. Wróbel MP, Marek B, Kajdaniuk D, et al. Metformin - a new old drug. Endokrynol Pol. 2017; 68(4): 482-496, doi: 10.5603/EP.2017.0050, indexed in Pubmed: 28819951.

9. UK Prospective Diabetes Study (UKPDS) Group. Effect of intensive blood-glucose control with metformin on complications in overweight patients with type 2 diabetes (UKPDS 34). Lancet. 1998; 352(9131): 854-865, indexed in Pubmed: 9742977.

10. Holman RR, Paul SK, Bethel MA, et al. 10-year follow-up of intensive glucose control in type 2 diabetes. N Engl J Med. 2008; 359(15): 1577-1589, doi: 10.1056/NEJMoa0806470, indexed in Pubmed: 18784090.

11. Eurich DT, Majumdar SR, McAlister FA, et al. Improved clinical outcomes associated with metformin in patients with diabetes and heart failure. Diabetes Care. 2005; 28(10): 2345-2351, doi: 10.2337/diacare.28.10.2345, indexed in Pubmed: 16186261

12. Andersson C, Olesen JB, Hansen PR, et al. Metformin treatment is associated with a low risk of mortality in diabetic patients with heart failure: a retrospective nationwide cohort study. Diabetologia. 2010; 53(12) 2546-2553, doi: 10.1007/s00125-010-1906-6, indexed in Pubmed: 20838985.

13. MasoudiFA, Inzucchi SE, Wang Y, et al Thiazolidinediones, metformin and outcomes in older patients with diabetes and heart failure: an observational study. Circulation. 2005; 111(5): 583-590, doi: 10.1161/01. CIR.0000154542.13412.B1, indexed in Pubmed: 15699279.

14. MacDonald MR, Eurich DT, Majumdar SR, et al. Treatment of type 2 diabetes and outcomes in patients with heart failure: a nested case-control study from the U.K. General Practice Research Database. Diabetes Care. 2010; 33(6): 1213-1218, doi: 10.2337/dc09-2227, indexed in Pubmed: 20299488.

15. Aguilar D, Chan W, Bozkurt B, et al. Metformin use and mortality in ambulatory patients with diabetes and heart failure. Circ Heart Fail. 2011; 4(1): 53-58, doi: 10.1161/CIRCHEARTFAILURE.110.952556, indexed in Pubmed: 20952583

16. Shah DD, Fonarow GC, Horwich TB. Metformin therapy and outcomes in patients with advanced systolic heart failure and diabetes. J Card Fail. 2010; 16(3): 200-206, doi: 10.1016/j.cardfail.2009.10.022, indexed in Pubmed: 20206893.

17. Wróbel M, Rokicka D, Zdrojewski T, et al. Metformin and cardiac injury after acute coronary syndrome in diabetic patients with no history of cardiovascular disease: data from the PL-ACS registry. Pol Arch Intern Med. 2020; 130(7-8): 708-710, doi: 10.20452/pamw.15475, indexed in Pubmed: 32621666.

18. Fácila L, Fabregat-Andrés Ó, Bertomeu V, et al. Metformin and risk of long-term mortality following an admission for acute heart failure. J Cardiovasc Med (Hagerstown). 2017; 18(2): 69-73, doi: 10.2459/JCM.0000000000000420, indexed in Pubmed: 27341193.

19. Seferović PM, Petrie MC, Filippatos GS, et al. Type 2 diabetes mellitus and heart failure: a position statement from the Heart Failure Association of the European Society of Cardiology. Eur J Heart Fail. 2018; 20(5): 853-872, doi: 10.1002/ejhf.1170, indexed in Pubmed: 29520964.

20. Rydén L, Grant PJ, Anker SD, et al. Authors/Task Force Members, ESC Committee for Practice Guidelines (CPG), Document Reviewers. ESC Guidelines on diabetes, pre-diabetes, and cardiovascular diseases developed in collaboration with the EASD: the Task Force on diabetes, pre-diabetes, and cardiovascular diseases of the European Society of Cardiology (ESC) and developed in collaboration with the European Association for the Study of Diabetes (EASD). Eur Heart J. 2013; 34(39): 3035-3087, doi: 10.1093/eurheartj/eht108, indexed in Pubmed: 23996285.

21. Grant PJ, Cosentino F, Cosentino F, et al. ESC Scientific Document Group. 2019 ESC Guidelines on diabetes, pre-diabetes, and cardiovascular diseases developed in collaboration with the EASD. Eur Heart J. 2020; 41(2): 255-323, doi: 10.1093/eurheartj/ehz486, indexed in Pubmed: 31497854.

22. Davies MJ, D'Alessio DA, Fradkin J, et al. Management of Hyperglycemia in Type 2 Diabetes, 2018. A Consensus Report by the American Diabetes Association (ADA) and the European Association for the Study of Diabetes (EASD). Diabetes Care. 2018; 41(12): 2669-2701, doi: 10.2337/dci18-0033, indexed in Pubmed: 30291106. 
23. Buse JB, Wexler DJ, Tsapas A, et al. 2019 Update to: Management of Hyperglycemia in Type 2 Diabetes, 2018. A Consensus Report by the American Diabetes Association (ADA) and the European Association for the Study of Diabetes (EASD). Diabetes Care. 2020; 43(2): 487-493, doi: 10.2337/dci19-0066, indexed in Pubmed: 31857443.

24. Eurich DT, Tsuyuki RT, Majumdar SR, et al. Metformin treatment in diabetes and heart failure: when academic equipoise meets clinical reality. Trials. 2009; 10: 12, doi: 10.1186/1745-6215-10-12, indexed in Pubmed: 19203392.

25. Pongwecharak J, Tengmeesri N, Malanusorn N, et al. Prescribing metformin in type 2 diabetes with a contraindication: prevalence and outcome. Pharm World Sci. 2009; 31(4): 481-486, doi: 10.1007/s11096-009-9303-2, indexed in Pubmed: 19462255.

26. Salvatore T, Pafundi PC, Marfella R, et al. Metformin lactic acidosis: Should we still be afraid? Diabetes Res Clin Pract. 2019; 157: 107879, doi: 10.1016/j.diabres.2019.107879, indexed in Pubmed: 31618624.

27. Federal Drug and Food Administration. Glucophage (metformin hydrochloride) label. Reference ID: 4079189. https://www.accessdata. fda.gov/drugsatfda_docs/label/2017/020357s037s039,021202s021s0231 bl.pdf (April 201)

28. Charakterystyka Produktu Leczniczego Glucophage $500 \mathrm{mg}$. http:// leki.urpl.gov.pl/files/43_Glucophage_500_mg_tabl_powl_500mg. pdf (13.01.2017).

29. Zinman B, Wanner C, Lachin JM, et al. EMPA-REG OUTCOME Investigators. Empagliflozin, Cardiovascular Outcomes, and Mortality in Type 2 Diabetes. N Engl J Med. 2015; 373(22): 2117-2128, doi: 10.1056/NEJMoa1504720, indexed in Pubmed: 26378978.

30. Figtree GA, Rådholm K, Neal B, et al. Canagliflozin and Heart Failure in Type 2 Diabetes Mellitus: Results From the CANVAS Program. Circulation. 2018; 138(5): 458-468, doi: 10.1161/CIRCULATIONAHA.118.034222, indexed in Pubmed: 29526832.

31. Packer M, Anker SD, Butler J, et al. EMPEROR-Reduced Trial Investigators. Cardiovascular and Renal Outcomes with Empagliflozin in Heart Failure. N Engl J Med. 2020; 383(15): 1413-1424, doi: 10.1056/NEJMoa2022190, indexed in Pubmed: 32865377. 